\title{
One-year mortality in displaced intracapsular hip fractures and associated risk: a report of Chinese-based fragility fracture registry
}

Simon Kwoon-Ho Chow ${ }^{1 \dagger}$, Jiang-hui Qin ${ }^{2 \dagger}$, Ronald Man-Yeung Wong ${ }^{1}$, Wai-Fan Yuen ${ }^{1}$, Wai-Kit Ngai ${ }^{3}$, Ning Tang ${ }^{1}$, Chor-Yin Lam ${ }^{4}$, Tak-Wing Lau', Kin-Bong Lee ${ }^{6}$, Kwai Ming Siu, Sze-Hung Wong ${ }^{8}$, Tracy Y. Zhu',

Wing-Hoi Cheung ${ }^{1 *}$ and Kwok-Sui Leung ${ }^{1}$

\begin{abstract}
Background: The purpose of this registry-based retrospective study was to investigate the risk factors related to one-year mortality in displaced intracapsular fragility hip fracture patients.

Methods: Patients were screened from the Fragility Fracture Registry. Inclusion criterion was displaced intracapsular hip fracture patients with atypical or pathological fractures excluded. One-year mortality was investigated against risk factors including age, gender, past medical history, pre-fracture mobility (PFM), pre-operation ASA grade, delayed surgery over $48 \mathrm{~h}$, post-surgical complications, and length of stay at acute orthopedic ward (LOS).
\end{abstract}

Results: A total of 1050 patients were included for further analysis. Gross one-year mortality was 14.9\%. One-year mortality was significantly higher in patients who received non-operative treatment and those who received surgery but delayed over $48 \mathrm{~h}$ after admission (both $p<0.001)$. Male gender $(\mathrm{OR}=2.708)$, advanced age $(\mathrm{OR}=1.359)$, higher risk ASA grades (III to $V)(O R=1.990)$, past history of gastrointestinal disease $(O R=1.671)$, and renal impairment $(O R=1$. 984) were related to higher one-year mortality. The mortality of patients in PFM grade 3 and LOS group 3 was significantly higher $(\mathrm{OR}=2.240$ and 1.722 , respectively).

Conclusions: Higher age, male gender, past gastrointestinal disease and renal impairment, ASA grade over 3, indoor confined pre-fracture ambulatory, and stay at hospital over 15 days were risk factors related to higher one-year mortality in surgically treated displaced intracapsular hip fracture patients. A multi-disciplinary approach is advised to patients identified with these risks factors and co-managed by orthopedic surgeons, geriatricians, and fracture liaison nurses.

Keywords: Fragility Fracture Registry, Intracapsular fragility hip fracture, One-year mortality, Risk factors

\section{Background}

Fragility fracture is a kind of fracture that occurs as a result of a low-energy trauma, such as a fall from a standing height or less, or even no identifiable trauma. It is the main adverse consequence of osteoporosis [1]. Osteoporosis-

\footnotetext{
*Correspondence: louis@ort.cuhk.edu.hk

'Simon Kwoon-Ho Chow and Jiang-hui Qin contributed equally to this work. 'Department of Orthopaedics and Traumatology, Prince of Wales Hospital, The Chinese University of Hong Kong, 5/F, Clinical Sciences Building, Shatin, New Territories, Hong Kong, SAR, China

Full list of author information is available at the end of the article
}

related fragility hip fracture is a worldwide public health problem, as it is significantly associated with morbidity and mortality [2, 3]. It causes an increasing burden on the healthcare system especially in the aging society $[4,5]$. Meanwhile, the incidences of osteoporosis and fragility fractures vary globally. According to Cooper's study, around $30 \%$ of the fragility hip fracture occurring worldwide were arisen in Asian populations and the incidence rates still keep rising predominately among Chinese and Malay subsets of the population [6]. Interestingly, the age-adjusted incidences in Hong Kong and Singapore increased from

(c) The Author(s). 2018 Open Access This article is distributed under the terms of the Creative Commons Attribution 4.0 International License (http://creativecommons.org/licenses/by/4.0/), which permits unrestricted use, distribution, and 
1960 s to 1980 s, synchronized with the modernization of the society $[7,8]$. This suggests that urbanization with contemporary changes in physical activities and nutrition may contribute to the increasing incidences of fragility hip fractures [6]. As the urbanization process predominantly happens in Asia especially in Mainland China, a study of agerelated, Chinese-based hip fracture in a developed Chinese society is of intriguing clinical implications.

Although the age-adjusted incidences of fragility hip fractures have become stable as healthcare quality improves [6], the gross incidences of fragility hip fractures keep steadily increasing because the society keeps aging and the mortality rate of these fractures was found to be generally high [9] . Compared to the annual $4 \%$ increase of mortality in hip fracture patients [10], the high mortality rate in the first year after fracture made this period most critical. Several factors have been identified related to the mortality of patients after surgery, such as increased age, male gender, ASA (American Society of Anesthesiologists) grade, types of surgery patients received, and walking ability before fracture [11, 12]. However, the results were controversial. For example, male gender was considered not related to the one-year mortality in the patients over 75 years old [11] but some other studies found it a major risk factor [13, 14]. In order to understand some population-specific risk factors that may contribute to increased mortality, a Fragility Fracture Registry was therefore established [9]. The objective of this study was to investigate the risk factors related to one-year mortality in displaced intracapsular fragility hip fracture patients, in which age, gender, pre-fracture mobility status, ASA grade, and length of stay at acute orthopedic ward were evaluated.

\section{Methods}

Patients were retrospectively screened from the Fragility Fracture Registry (FFR, www.ffr.hk) database, which collected data of 2914 cases of fragility hip fracture patients who were admitted during 2012 to six major public hospitals under the management of the Hospital Authority of Hong Kong (www.ha.org.hk) [9]. Ethical approvals were obtained from the Research Ethics Committee (REC) of all six hospitals. Consent from subjects was not required. The study was done in accordance with the principles outlined in the Declaration of Helsinki. All patients fit the definition of fragility fracture as mentioned above [1] with age not less than 50 years old who sustained a hip fracture falling from standing height as previously described [9]. Only patients with displaced intracapsular hip fracture were included in this study. Cases of atypical or pathological fractures were excluded. Information including age, gender, pre-fracture medical history, previous fracture history, pre-fracture mobility status (PFM), pre-operation ASA (American Society of Anesthesiologists) grade, type of operation, surgical status at $48 \mathrm{~h}$ after admission, length of stay at acute orthopedic ward (LOS), and post-surgical complications were recorded.

The pre-fracture medical history record including previous episodes of arthritis, osteoporosis, cerebrovascular disease, dementia, depression, diabetes mellitus, gastrointestinal diseases, heart diseases, hypertension, hypotension, renal impairment, respiratory diseases, Parkinson's disease, visual impairment, and other miscellaneous options. The post-surgical complications recorded the occurrence of deep vein thrombosis, wound infection, postoperative delirium, pressure sore, retention of urine, and other miscellaneous options. Time-to-surgery was calculated from the time of first presentation in hospital to the time of operation.

According to the ambulation status before fracture, PFM is divided into 3 grades: grade 1 (fully ambulatory), grade 2 (ambulatory with aids), and grade 3 (indoor confined).

According to the ASA score, patients were divided into 2 groups. The lower risk group (ASA grade I and II) included patients with healthy or mild systemic disease. The higher risk group (ASA grade III to V) included patients with severe non-incapacitating systemic disease and severe incapacitating systemic disease with a constant threat to life. No patient with ASA grade V was present in this study.

The impact of LOS on mortality of fragility hip fracture was studied extensively, but the grading standards vary among different studies $[15,16]$. In this study, we consider LOS from the 25 percentiles (6.68 days) to 75 percentiles (15.09 days) as normal, the first quadrant as early-discharge patient and the last quadrant as delay-discharge patient. According to this standard, the patients were divided into 3 groups: LOS 1 ( $\leq 7$ days); LOS 2 ( $7-15$ days); LOS 3 (> 15 days).

\section{Statistical analyses}

All data were managed using Research Electronic Data Capture (REDCap; Vanderbilt University) hosted in The Chinese University of Hong Kong [17]. Independent two-sample $t$ test and Pearson's Chi-square test were performed to compare age, gender, PFM, LOS, and one-year mortality between patients who received non-operative and surgical treatments. Potential risk factors related to the post-fracture one-year mortality were first screened by using Pearson's Chi-square test (univariate). Risk factors including gender, age, PFM, LOS, ASA grade, delayed surgery, past medical history of respiratory disease, heart disease, hypertension, gastrointestinal disease, renal impairment, and post-surgical wound infection were further investigated by using logistic regression (multivariate). As delayed surgery was significantly associated with LOS and ASA grade, it was further excluded from the 
model to avoid collinearity. Female gender, PFM grade 1, low risk ASA group, and LOS group 2 were set as reference groups, respectively, and odds of mortality associated with age was presented as per decade increase. All statistical analyses were performed by using SPSS statistical software version 20.0 (IBM, NY, USA). A $p$ value less than 0.05 was considered as statistically significant.

\section{Results}

One thousand and sixty-one patients were captured during the study period. The majority of fractures (965 cases, $91 \%)$ were caused by fall. The leading causes included slip and fall (73.4\%), fall from furniture (8.2\%), and tripped by obstacles (5.8\%). Eleven patients were excluded from the study due to missing data (one without one-year mortality, one without type of treatment, six without PFM, and three without LOS).

A total of 1050 patients (574 left hip fracture and 476 right hip fracture) were included for analysis in this study, among which $94.3 \%$ received surgical treatment and $5.7 \%$ received non-operative treatment. Table 1 shows age, gender, PFM, LOS, and one-year mortality of the patients by non-operative and surgical treatments. The gross one-year mortality of displaced intracapsular hip fracture was $14.9 \%$, and mortality was significantly higher in the patients who received non-operative treatment than those who received surgical treatment $(53.3 \%$ vs. $12.5 \%, p<0.001$ ). Age did not differ significantly between non-operative and surgical groups $(p=0.25)$, while the patients who received non-operative treatment were significantly more likely to be male $(p=0.028)$, in PFM grade $3(p=0.001)$, and have a longer LOS ( $p=$ 0.012 ). Previous episodes of cerebrovascular accidents $(48.3 \%$ vs. $21.5 \%, p<0.001)$ and heart diseases $(58.3 \%$ vs.

Table 1 Demographic data on patients in the conservative and surgically treated group

\begin{tabular}{llll}
\hline Demographic variables & & Conservative & Surgical \\
\hline Number of patients & & $60(5.7 \%)$ & $990(94.3 \%)$ \\
Gender & Male & $26(43.3 \%)$ & $288(29.1 \%)$ \\
Age, years & Female & $34(56.7 \%)$ & $702(70.9 \%)$ \\
& Mean \pm SD & $82.5 \pm 10.0$ & $81.1 \pm 8.9$ \\
Pre-fracture mobility (PFM) & Range & $58-100$ & $50-104$ \\
& Grade 1 & $16(26.7 \%)$ & $399(40.3 \%)$ \\
& Grade 2 & $22(36.7 \%)$ & $431(43.5 \%)$ \\
Length of stay (LOS) & Grade 3 & $22(36.7 \%)$ & $160(16.2 \%)$ \\
& Quadrant 1 & $24(40.0 \%)$ & $277(28.0 \%)$ \\
& Quadrant 2 & $22(36.7 \%)$ & $467(47.2 \%)$ \\
& Quadrant 3 & $6(10.0 \%)$ & $185(18.7 \%)$ \\
One-year mortality & Quadrant 4 & $8(13.3 \%)$ & $61(6.2 \%)$ \\
& & $32(53.3 \%)$ & $124(12.5 \%)$ \\
\hline
\end{tabular}

$29.2 \%, \mathrm{p}<0.001$ ) were found significantly more common in the patients who received non-operative treatment.

Analyses were performed to identify risk factors for one-year mortality in the patients who received surgical treatment. Ten patients were excluded from the analyses due to lack of ASA grade. Analyses were performed in 980 patients. A total of 870 patients (88.8\%) received unipolar hemiarthroplasty, which was the most commonly used surgical procedure. Of these patients, 768 received uncemented and 102 received cemented hemiarthroplasty. The use of cement during hemiarthroplasty showed no effect on the one-year mortality $(12.7 \%$ in cemented who died vs. $13.0 \%$ in uncemented patients, $p=$ $0.94)$. Five hundred and eighty-two patients (59.4\%) received surgery within $48 \mathrm{~h}$ after admission while surgeries were delayed in 398 patients (40.6\%). The most common reason of delayed surgery was prolonged medical review or stabilization of the patients (250 patients, 62.8\%). One-year mortality was significantly higher in the patients with delayed surgery $(54.5 \%$ vs. $38.6 \%, p=0.001)$.

Logistic regression was performed to identify the risk factors (gender, increased age of every 10 years, PFM, LOS, ASA grade, past medical history of respiratory disease, heart disease, hypertension, gastrointestinal disease, renal impairment, and post-surgical wound infection) of one-year mortality (Table 2). Time-to-surgery was excluded from logistic regression analysis to avoid collinearity because it was significantly correlated with LOS and ASA score (both $p<$ $0.001)$. Male gender was a strong risk factor of oneyear mortality, with odds of mortality almost tripled in males than in females $(\mathrm{OR}=2.708$, 95\% CI $1.766-$ $4.151, \mathrm{p}<0.001)$. One-year mortality significantly increased with age, with each decade increase in age associated with $35.9 \%$ increase in mortality $(\mathrm{OR}=1.359$, 95\% CI 1.041-1.773, $p=0.024)$. Patients with higher risk ASA score group had significantly higher one-year mortality than those with lower risk ASA score (OR = 1.990, 95\% CI 1.182-3.349, $p=0.010$ ). Mortality did not differ significantly between patients with PFM grade 2 and grade $1(p=0.253)$, but was significantly higher in the patients with grade 3 than those with grade $1(\mathrm{OR}=2.240,95 \%$ CI $1.229-4.085, p=0.008)$. One-year mortality was similar between the patients who were fully ambulatory and those were ambulatory with aids, while patients who were indoor confined had 2.2 times higher risk of one-year mortality. Compared to the patients of LOS group 2, one-year mortality of group 3 was significantly higher $(\mathrm{OR}=1.772$, 95\% CI 1.075-2.758, $\mathrm{p}=0.024$ ), but no difference was observed between group 2 and group 1, suggesting that risk of one-year mortality was higher only when LOS was beyond 15 days. Previous history of gastrointestinal disease $(\mathrm{OR}=1.671,95 \% \mathrm{CI} 1.084-2.575, p$ 
Table 2 Mortality risk for different risk factors including pre and post-operational assessments and medical history

\begin{tabular}{|c|c|c|c|c|c|}
\hline Risk factors & Dead & Alive & OR & $95 \% \mathrm{Cl}$ & $p$ value \\
\hline Number of patients & 121 & 859 & - & - & - \\
\hline \multicolumn{6}{|l|}{ Gender } \\
\hline Male & $59(48.8 \%)$ & $227(26.4 \%)$ & 2.708 & $1.766-4.151$ & $<0.001$ \\
\hline Female* & $62(51.2 \%)$ & $632(73.6 \%)$ & - & - & - \\
\hline Age, years & & & $1.359^{\#}$ & $1.041-1.773$ & 0.024 \\
\hline Mean \pm SD & $8.36 \pm 0.80$ & $8.08 \pm 0.89$ & & & \\
\hline Range & $5.9-10.0$ & $5.0-10.4$ & & & \\
\hline \multicolumn{6}{|l|}{ Pre-fracture mobility } \\
\hline Grade 2 & $58(47.9 \%)$ & $367(42.7 \%)$ & 1.352 & $0.806-2.270$ & 0.253 \\
\hline Grade 3 & $34(28.1 \%)$ & $125(14.6 \%)$ & 2.240 & $1.229-4.085$ & 0.008 \\
\hline Grade $1^{*}$ & $29(24.0 \%)$ & $367(42.7 \%)$ & - & - & - \\
\hline \multicolumn{6}{|l|}{ ASA Grade } \\
\hline High risk (III to V) & 97 (80.2\%) & $433(50.4 \%)$ & 1.990 & $1.182-3.349$ & 0.010 \\
\hline Low risk (I and II)* & $24(19.8 \%)$ & $426(49.6 \%)$ & - & - & - \\
\hline \multicolumn{6}{|l|}{ Length of stay (LOS) } \\
\hline Group 1 ( $\leq 7$ days) & $21(17.4 \%)$ & 255 (29.7\%) & 0.683 & $0.385-1.211$ & 0.192 \\
\hline Group 3 (> 15 days) & $53(43.8 \%)$ & $191(22.2 \%)$ & 1.722 & $1.075-2.758$ & 0.024 \\
\hline Group 2 (7-15 days)* & 47 (38.8\%) & $413(48.1 \%)$ & - & - & - \\
\hline \multicolumn{6}{|l|}{ Past respiratory disease } \\
\hline Yes & $31(25.6 \%)$ & $217(25.3 \%)$ & 1.584 & $0.965-2.600$ & 0.069 \\
\hline $\mathrm{No}^{*}$ & $90(74.4 \%)$ & $642(74.7 \%)$ & - & - & - \\
\hline \multicolumn{6}{|l|}{ Past heart disease } \\
\hline Yes & $31(25.6 \%)$ & $217(25.3 \%)$ & 1.382 & $0.891-2.145$ & 0.149 \\
\hline $\mathrm{No}^{*}$ & $90(74.4 \%)$ & $642(74.7 \%)$ & - & - & - \\
\hline \multicolumn{6}{|c|}{ Past history of hypertension } \\
\hline Yes & $31(25.6 \%)$ & $217(25.3 \%)$ & 1.172 & $0.725-1.896$ & 0.517 \\
\hline $\mathrm{No}^{*}$ & $90(74.4 \%)$ & $642(74.7 \%)$ & - & - & - \\
\hline \multicolumn{6}{|c|}{ Past gastrointestinal disease } \\
\hline Yes & $31(25.6 \%)$ & $217(25.3 \%)$ & 1.671 & $1.084-2.575$ & 0.020 \\
\hline $\mathrm{No}^{*}$ & $90(74.4 \%)$ & $642(74.7 \%)$ & - & - & - \\
\hline \multicolumn{6}{|l|}{ Past renal impairment } \\
\hline Yes & $31(25.6 \%)$ & $217(25.3 \%)$ & 1.984 & $1.201-3.277$ & 0.007 \\
\hline $\mathrm{No}^{*}$ & $90(74.4 \%)$ & $642(74.7 \%)$ & - & - & - \\
\hline \multicolumn{6}{|c|}{ Post-surgical wound infection } \\
\hline Yes & $66(54.5 \%)$ & $332(38.6 \%)$ & 1.920 & $0.826-4.460$ & 0.129 \\
\hline $\mathrm{No}^{*}$ & $55(45.5 \%)$ & $527(61.4 \%)$ & - & - & - \\
\hline
\end{tabular}

\#Per decade increase in age

*Reference group

$=0.020)$ and renal impairment $(\mathrm{OR}=1.984,95 \% \mathrm{CI}$ 1.201-3.277, $p=0.007$ ) were significantly associated with increased one-year mortality. Past history of respiratory disease, heart disease, hypertension, and post-surgical wound infection did not show significant impact on one-year mortality $(p=0.069,0.149,0.517$, and 0.129 , respectively).

\section{Discussion}

In this study, we investigated one-year mortality and its related risk factors in the patients with displaced intracapsular hip fracture in a Chinese-based population. We found that patients who received non-operative management had higher mortality than those who received surgical treatment. However, direct evaluation of pre-surgical 
health condition of these patients was not feasible due to lack of ASA scores in the non-operative treated patients, yet their poorer pre-fracture mobility and longer length of stay at acute orthopedic ward still indicated poorer general health condition and more in-hospital comorbidities. These patients also had higher incidence of previous episode of cerebrovascular accident and heart disease. A study on Korean population also reported associations between mortality and chronic illnesses including hypertension, diabetes, dyslipidemia, ischemic heart disease, and stroke [18]. Hence, poor general health condition was the major factor correlated to the patients' selection of non-operative management, while age and gender showed no effect.

In the patients who received surgical treatment, higher mortality in male was observed. This result was in agreement with a similar previous study [14] that is also associated with advanced age similar to a previous study [19]. Previous studies found that male patients with hip fracture usually had poorer health status before surgery with worse ASA grades [13] and pre-existing cerebrovascular and pulmonary diseases were associated to more post-surgical comorbidities such as developing pneumonia and cardiac complications [13, 20]. However, in our multivariate logistic regression analyses, male gender remained significant after adjustment of ASA grade and past medical history of comorbidities, suggesting that, at least in part, male gender per se led to higher one-year mortality of hip fracture. However, due to different inclusion criteria and varied sample sizes among studies, direct comparison with previous studies must be taken with caution. It has also been reported that gender was not related to the mortality in hip fracture patients over 75 years old [11].

Pre-fracture mobility reflects the general condition of patient's health and related to the outcomes of surgery. Previous studies reported that walking ability before hip fracture could predict greater long-term survival [12] and affect the short-term and long-term mortality in hip fracture patients [20]. Similar findings were observed in this study. Patients with poorer ambulatory status before fracture was found related to higher one-year mortality rate. Length of hospital stay was correlated to both short-term and long-term mortality in surgically treated hip fracture patients. Cohort studies from northern Europe showed that LOS of less than 10 days was associated to both better short-term and long-term survival $[16,21]$. However, LOS of less than 5 days was found to be associated with reduced early mortality rates when comparing to LOS of 11-14 days in the USA [15]. Our study found that there was no difference in one-year mortality between early discharged patients with LOS less than 7 days and those with LOS between 7 to 15 days. On the other hand, LOS over 15 days was found correlated to increased short-term mortality in the USA [15] and higher one-year mortality in our study. In
Denmark, prolonged LOS over 20 days was also associated with long-term mortality [21]. The lack of difference between short and intermediate LOS in this study may be due to a lack of interdisciplinary management. Since our current study also revealed that history of geriatric medical conditions like gastrointestinal and renal diseases substantially increased the risks of one-year mortality, and also shown by our previous report that only $3.5 \%$ of hip-fracture patients received geri-orthopedic co-management [9]. Furthermore, decreased muscle strength and vitamin D deficiency in geriatric hip fracture patients were also reported to increase mortality rates in the Spanish FONDA cohort [22]. Therefore, a multi-disciplinary management approach [23, 24] (by orthopedic surgeons, geriatricians, and fracture liaison nurses) is strongly advised to patients identified with these risks factors (in particular, pre-fracture mobility and previous medical conditions) in order to proactively work towards improving the survival rate after an intracapsular hip fracture event. More detailed investigation is suggested to dissect the relative risks in various medically compromised groups.

There are limitations in this study. The power of this study is limited to the intracapsular neck of femur fractures that generalization to hip fracture shall be taken with caution. DXA data were not available from the public healthcare system for further analysis. Cognitive status was closely related to the prognosis of patients with hip fracture [25, 26]. However, it was not analyzed in this study due to the relatively small number of patients who received cognitive function assessments, and the influence of medication to the fracture was not studied due to similar reasons.

\section{Conclusion}

In the patients with displaced intracapsular hip fracture, one-year mortality was significantly higher in the patients who received non-operative treatment than those who received surgical treatment. Risk factors related to one-year mortality in surgically treated displaced intracapsular hip fracture patients included older age, male gender, ASA grade over 3, an indoor-confined pre-fracture mobility status, and stay at hospital over 15 days. A multi-disciplinary approach is advised to patients identified with these risks factors and co-managed by orthopedic surgeons, geriatricians, and fracture liaison nurses.

\section{Abbreviations}

ASA: American Society of Anesthesiologists; Cl: Confidence interval; FFR: Fragility Fracture Registry; LOS: Length of stay; OR: Odds ratio; PFM: Prefracture mobility; REDCap: Research Electronic Data Capture

\section{Acknowledgements}

We thank all clinical staff members and nurses who have contributed to data collection and validation in the six participating hospitals in the Fragility 
Fracture Registry. This study is partially supported by the National Natural Science Foundation of China (NSFC, 81472097).

\section{Availability of data and materials}

Data and patient information is not publically available, and access to data by research personnel is strictly adhered to the ethical approvals and relevant regulations obtained from the six hospitals.

\section{Authors' contributions}

WHC and KSL contributed to concept and design. SKC, JHQ, WFY, WKN, NT, CYL, TWL, KBL, KMS, SHW, and KSL contributed to data acquisition. SKC, JHQ, RMYW, and TYZ contributed to analysis and interpretation of data. SKC, JHQ, RMYW, TYZ, and WHC contributed to the drafting of the manuscript. JHQ, RMYW, WKN, NT, CYL, TWL, KBL, KMS, SHW, TYZ, WHC, and KSL contributed to critical revision for intellectual content. All authors read and approved the final manuscript.

\section{Ethics approval and consent to participate}

Ethical approvals were obtained from the Research Ethics Committee (REC) of all six hospitals (reference numbers: KW/EX-13-094(65-06(CMC)) at the Caritas Medical Centre, KW/EX-13-095(65-06(PMH)) at the Princess Margaret Hospital, CT-209/2013 at the Prince of Wales Hospital, KC/KE-13-0106/ER-2 at the Queen Elizabeth Hospital, UW-13-379 at the Queen Mary Hospital, and NTWC/CREC/1170/13 at the Tuen Mun Hospital). The study was done in accordance with the principles outlined in the Declaration of Helsinki [1].

\section{Consent for publication}

Not applicable.

\section{Competing interests}

The authors declare that they have no competing interests.

\section{Publisher's Note}

Springer Nature remains neutral with regard to jurisdictional claims in published maps and institutional affiliations.

\section{Author details}

'Department of Orthopaedics and Traumatology, Prince of Wales Hospital, The Chinese University of Hong Kong, 5/F, Clinical Sciences Building, Shatin, New Territories, Hong Kong, SAR, China. ${ }^{2}$ Department of Sports Medicine and Adult Reconstructive Surgery, Drum Tower Hospital, School of Medicine, Nanjing University, Nanjing, China. ${ }^{3}$ Department of Orthopaedics and Traumatology, North District Hospital, Sheung Shui, Hong Kong. ${ }^{4}$ Department of Orthopaedics and Traumatology, Tuen Mun Hospital, Tuen Mun, Hong Kong. ${ }^{5}$ Department of Orthopaedics and Traumatology, Queen Mary Hospital, Pok Fu Lam, Hong Kong. ${ }^{6}$ Department of Orthopaedics and Traumatology, Queen Elizabeth Hospital, Jordan, Hong Kong. ${ }^{7}$ Department of Orthopaedics and Traumatology, Princess Margaret Hospital, Kwai Chung Hong Kong. ${ }^{8}$ Department of Acute and Rehabilitative Orthopaedics and Traumatology, Caritas Medical Centre, Sham Shui Po, Hong Kong.

Received: 1 June 2018 Accepted: 3 September 2018

\section{Published online: 14 September 2018}

\section{References}

1. Brown JP, Josse RG. Scientific Advisory Council of the Osteoporosis Society of C: 2002 clinical practice guidelines for the diagnosis and management of osteoporosis in Canada. CMAJ. 2002;167:S1-34.

2. Magaziner J, Lydick E, Hawkes W, Fox KM, Zimmerman SI, Epstein RS, Hebel $J R$. Excess mortality attributable to hip fracture in white women aged 70 years and older. Am J Public Health. 1997;87:1630-6.

3. Omsland TK, Emaus N, Tell GS, Magnus JH, Ahmed LA, Holvik K, Center J, Forsmo S, Gjesdal CG, Schei B, et al. Mortality following the first hip fracture in Norwegian women and men (1999-2008). A NOREPOS study. Bone. 2014; 63:81-6.

4. Brainsky A, Glick H, Lydick E, Epstein R, Fox KM, Hawkes W, Kashner TM, Zimmerman SI, Magaziner J. The economic cost of hip fractures in community-dwelling older adults: a prospective study. J Am Geriatr Soc. 1997:45:281-7

5. Johnell O, Kanis J. Epidemiology of osteoporotic fractures. Osteoporos Int. 2005;16(Suppl 2):S3-7.
6. Cooper C, Cole ZA, Holroyd CR, Earl SC, Harvey NC, Dennison EM, Melton LJ, Cummings SR, Kanis JA. Epidemiology ICWGoF: secular trends in the incidence of hip and other osteoporotic fractures. Osteoporos Int. 2011;22:1277-88.

7. Lau EM, Cooper C, Fung H, Lam D, Tsang KK. Hip fracture in Hong Kong over the last decade--a comparison with the UK. J Public Health Med. 1999; 21:249-50.

8. Koh LK, Saw SM, Lee JJ, Leong KH, Lee J. National Working Committee on O: hip fracture incidence rates in Singapore 1991-1998. Osteoporos Int. 2001;12:311-8.

9. Leung KS, Yuen WF, Ngai WK, Lam CY, Lau TW, Lee KB, Siu KM, Tang N, Wong $\mathrm{SH}$, Cheung WH. How well are we managing fragility hip fractures? A narrative report on the review with the attempt to setup a Fragility Fracture Registry in Hong Kong. Hong Kong Med J. 2017;23:264-71.

10. Paksima N, Koval KJ, Aharanoff G, Walsh M, Kubiak EN, Zuckerman JD, Egol KA. Predictors of mortality after hip fracture: a 10-year prospective study. Bull NYU Hosp Jt Dis. 2008;66:111-7.

11. Karademir G, Bilgin Y, Ersen A, Polat G, Buget MI, Demirel M, Balci HI. Hip fractures in patients older than 75 years old: retrospective analysis for prognostic factors. Int I Surg. 2015;24:101-4.

12. Iosifidis M, lliopoulos E, Panagiotou A, Apostolidis K, Traios S, Giantsis G. Walking ability before and after a hip fracture in elderly predict greater long-term survivorship. J Orthop Sci. 2016;21:48-52.

13. Ekstrom W, Samuelsson B, Ponzer S, Cederholm T, Thorngren KG, Hedstrom M. Sex effects on short-term complications after hip fracture: a prospective cohort study. Clin Interv Aging. 2015:10:1259-66.

14. Man LP, Ho AW, Wong SH. Excess mortality for operated geriatric hip fracture in Hong Kong. Hong Kong Med J. 2016;22:6-10.

15. Nikkel LE, Kates SL, Schreck M, Maceroli M, Mahmood B, Elfar JC. Length of hospital stay after hip fracture and risk of early mortality after discharge in New York state: retrospective cohort study. BMJ. 2015;351:h6246.

16. Nordstrom P, Gustafson Y, Michaelsson K, Nordstrom A. Length of hospital stay after hip fracture and short term risk of death after discharge: a total cohort study in Sweden. BMJ. 2015;350:h696.

17. Harris PA, Taylor R, Thielke R, Payne J, Gonzalez N, Conde JG. Research electronic data capture (REDCap)--a metadata-driven methodology and workflow process for providing translational research informatics support. J Biomed Inform. 2009;42:377-81.

18. Choi HG, Lee YB, Rhyu SH, Kwon BC, Lee JK. Mortality and cause of death postoperatively in patients with a hip fracture: a national cohort longitudinal follow-up study. Bone Joint J. 2018;100B:436-42.

19. Dokladalova P, Majernicek M, Vaculik J, Kubes R, Schwarz O, Dungl P. Results of surgically treated patients for hip fracture - complications, mortality. Acta Chir Orthop Traumatol Cech. 2017;84:424-30.

20. Pioli G, Lauretani F, Pellicciotti F, Pignedoli P, Bendini C, Davoli ML, Martini E, Zagatti A, Giordano A, Nardelli A, et al. Modifiable and non-modifiable risk factors affecting walking recovery after hip fracture. Osteoporos Int. 2016; 27(6):2009-16.

21. Lund CA, Moller AM, Wetterslev J, Lundstrom LH. Organizational factors and long-term mortality after hip fracture surgery. A cohort study of 6143 consecutive patients undergoing hip fracture surgery. PLoS One. 2014;9: e99308.

22. Menendez-Colino R, Alarcon T, Gotor P, Queipo R, Ramirez-Martin R, Otero A, Gonzalez-Montalvo Jl. Baseline and pre-operative 1-year mortality risk factors in a cohort of 509 hip fracture patients consecutively admitted to a co-managed orthogeriatric unit (FONDA cohort). Injury. 2018:49:656-61.

23. Shenouda M, Silk Z, Radha S, Bouanem E, Radford W. The introduction of a multidisciplinary hip fracture pathway to optimise patient care and reduce mortality: a prospective audit of 161 patients. Open Orthop J. 2017;11:309-15.

24. Wallace R, Angus LDG, Munnangi S, Shukry S, DiGiacomo JC, Ruotolo C. Improved outcomes following implementation of a multidisciplinary care pathway for elderly hip fractures. Aging Clin Exp Res. 2018; https://doi.org/ 10.1007/s40520-018-0952-7.

25. Sodergvist A, Ekstrom W, Ponzer S, Pettersson H, Cederholm T, Dalen N, Hedstrom M, Tidermark J. : prediction of mortality in elderly patients with hip fractures: a two-year prospective study of 1,944 patients. Gerontology. 2009:55:496-504.

26. Smith T, Pelpola K, Ball M, Ong A, Myint PK. Pre-operative indicators for mortality following hip fracture surgery: a systematic review and metaanalysis. Age Ageing. 2014;43:464-71. 